and related enzymes activities. Cell migration was measured by Boyden chamber. S1P and Sphk-1,2 was quantitatively measured both by HPLC-fluorescence detection after OPA derivatization and by LC-MS/MS system. HDAC activity assay kit (Cayman Co) was used. Sphk mRNA level was measured by qRT-PCR using $\Delta \Delta \mathrm{Ct}$ method. Antibodies for PKC isoform was obtained from Cell Science Co. and detected using western blot. Decursin inhibited sphingosine kinase (Sphk) induced angiogenic processes in vitro, including cell proliferation, migration of human umbilical vein endothelial cells (HUVEC). Interestingly, Sphk-1 activity was significantly decreased by $43 \%$ compared to control, while Sphk-II activity was 1.6 fold increased by decursin treatment. The S1P in cells and in cultured media was decreased dose-dependently by decursin, indicating that the reduced synthesis of angiogenic lipid mediator S1P which binding to S1P receptors (S1PRs) is essential to transmit S1P-S1PR axis signaling for angiogenesis. Decursin specifically reduced Rac-1.The increased Sphk-II activity and thus S1P production in nuclear fraction blocked histone deacetylase (HDAC) activity. Indeed, the mRNA expression of Sphk-II was 3-fold increased by decursin. Sphingosine level was also increased in 2-fold. Decursin treatment enhanced the migration of Sphk-1 and PKC $\alpha$ into nuclear membrane. The expression of $\mathrm{PKC} \alpha$ and $\mathrm{PKC} \eta$ (eta) were decreased while other PKC isoforms were not changed. Our data suggests that decursin have a potent anti-angiogenic property via S1PR-S1P axis by regulating both SPHK-I and -II activites and thus reducing S1P synthesis and release.

\title{
ISOLATION OF GELATIN HYDROLYSATES EFFECTIVE ON BONE FORMATION AND RESORPTION
}

\section{(ㄷ Leem Kang-Hyun ${ }^{1}$, Kim Hye Kyung ${ }^{2}$}

${ }^{1}$ College of Oriental Medicine, Semyung University, Jechon, Chungbuk 390-711, South Korea

${ }^{2}$ Department of Food and Biotechnology, Hanseo University, Seosan, Chungnam 356-706, South Korea

Gelatin is a mixture of polypeptides obtained by partial hydrolysis of collagens extracted from connective tissues of animals. The aim of this study was to provide the gelatin hydrolysate $(\mathrm{GH})$ which is effective on bone formation. Porcine skin gelatin was hydrolyzed by manipulating two reaction parameters; enzyme combination and reaction time. Fourcombinations ofenzymes(alcalase+protamex, protamex+flavourzyme, flavourzyme+alcalase and alcalase+protamex+flavourzyme) and 3 reaction times $(4,12$, and $24 \mathrm{~h}$ ) were examined. The resultant $12 \mathrm{GHs}$ were fractionated into 3 ranges of molecular weight (total, $>3 \mathrm{kDa}$, and $<3 \mathrm{kDa}$ ), and 36 various $\mathrm{GH}$ were obtained. An in vitro study on bone formation was carried out in osteoblast-like MG63 cell proliferation and opti- mal hydrolysis condition for the maximal bone formation activity was selected. The enzyme combination of protamex+flavourzyme provided the $\mathrm{GH}$ with the highest activity. Higher bone formation activity was observed in small molecular weight ( $\angle 3 \mathrm{kDa}) \mathrm{GH}$ at reaction time of 4 hour. The effects of selected $\mathrm{GH}$ on bone resorption were determined in bone marrow-derived osteoclasts cells driven by RANKL and M-CSF. GH suppressed the formation of TRAP-positive osteoclasts. Furthermore, RANKL induced TRAP activity was greatly inhibited by GH treatment. The effects on bone formation and resorption were not observed with gelatin treatment. These results suggest that $\mathrm{GH}$ isolated in this study is a promising agent for the prevention and treatment of bone loss.

\section{INTESTINAL ABSORPTION AND PRESYSTEMIC ELIMINATION OF VARIOUS CHEMICAL CONSTITUENTS PRESENT IN GBE50 EXTRACT, A STANDARDIZED EXTRACT OF GINKGO BILOBA LEAVES}

\section{(C) Li Chuan, Li Li, Zhao Yuansheng, Du Feifei, Yang Junling, Xu Fang, Niu Wei, Ren Yaohui}

Shanghai Institute of Materia Medica, Chinese Academy of Sciences, Shanghai, China

The nature and level of systemic exposure to the active herbal constituents will profoundly affect their effects at action sites, which is fundamental in understanding their roles in the overall beneficial effects of an herbal medicine. The objective of this study is to gain a full picture of the systemic exposure to various putatively active ginkgo constituents after p. o. administration of GBE50 extract, a standardized extract of Ginkgo biloba 